University of Windsor

Scholarship at UWindsor

Fall 2009

\title{
Production costs, scope economies, and multi-client outsourcing under quantity competition
}

\author{
Debing Ni \\ Kevin W. Li Dr. \\ University of Windsor \\ Xiaowo Tang
}

Follow this and additional works at: https://scholar.uwindsor.ca/odettepub

Part of the Business Commons

\section{Recommended Citation}

Ni, Debing; Li, Kevin W. Dr.; and Tang, Xiaowo. (2009). Production costs, scope economies, and multi-client outsourcing under quantity competition. International Journal of Production Economics, 121 (1), 130-140. https://scholar.uwindsor.ca/odettepub/63

This Article is brought to you for free and open access by the Odette School of Business at Scholarship at UWindsor. It has been accepted for inclusion in Odette School of Business Publications by an authorized administrator of Scholarship at UWindsor. For more information, please contact scholarship@uwindsor.ca. 


\title{
Production Costs, Scope Economies, and Multi-Client Outsourcing under Quantity Competition
}

\author{
Debing $\mathrm{Ni}^{1}$, Kevin W. $\mathrm{Li}^{2 *}$, Xiaowo Tang ${ }^{1}$ \\ 1 School of Management, University of Electronic Science and Technology of China, Chengdu, Sichuan, P. R. China, 610054 \\ 2 Odette School of Business, University of Windsor, Windsor, Ontario N9B 3P4, Canada,
}

\begin{abstract}
Two game models are developed based on production costs and scope economies to investigate the widely observed multi-client outsourcing (MCO) phenomenon. Analytical results demonstrate that outsourcers' high in-house production costs and the advantage of scope economies motivate firms to outsource collectively to an independent vendor. Under certain conditions, if both firms make their outsourcing decisions simultaneously, collective outsourcing is one of the two equilibria; if both firms make decisions sequentially, collective outsourcing becomes the unique equilibrium. Furthermore, the comparative statics of the critical degree of scope economies are examined for the occurrence of MCO with regard to diverse market parameters. Finally, it is proved that market prices decrease as the degree of scope economies increases when MCO occurs. This research helps explain some widely observed phenomena such as malls, supply chain cities, and the China price.
\end{abstract}

Keywords: scope economies, multi-client outsourcing, game models, subgame perfect equilibrium

\section{Introduction}

A value chain can usually be divided into several components such as design, manufacturing and marketing departments, with each handling a special (operational) function. However, it is not necessary for a firm to own all these functional departments. Instead, the firm may outsource one or more of these functions to an outside vendor. When two or more firms outsource to a same vendor, MCO (one vendor vs multiple clients) is observed (Sharma and Yetton 1996), which is sometimes referred to as co-sourcing (e.g. Gallivan and Oh 1999). Without causing confusion, we always use "firms" to represent companies that seek potential outsourcing opportunities and let "vendor" denote the company that provides outsourcing services.

MCO is widely observed in this era of increasingly competitive and globalizing economies. For example, firms in different industries turn their information systems over to relatively few computer companies such as IBM (Debbern et al. 2004), and more and more firms now outsource their logistics needs, resulting in the rapid growing of the third party logistics business (Berglund et al., 1999; Steffansson, 2002). Recently, the newly observed supply chain cities, located in Guangdong, China, can also be viewed as an outcome of MCO where several world-renowned apparel companies such as Polo Ralph Lauren, Liz Claiborne and Dillard's, and Fast Retailing outsource their production functions to Luen Thai Holdings Ltd. (Kahn 2004; Kusterbeck 2005). Even at the macro level, the observation that many multi-national companies in developed countries are moving their manufacturing facilities to developing countries such as China can also be treated as MCO.

Academia, business professionals, and even government officials have been extensively discussing the increasing trend of outsourcing manufacturing to China. As a direct consequence, products labeled both "Made in China" and a western brand are widely available in the marketplace and usually sold at a much lower price. The lower price is coined as "the China price” by Engardio et al. (2004) in a Business Week's special report. The China price implies that U.S. companies that manufacture in the United States must

\footnotetext{
* Correspondence author, phone: 1-519-253-3000 ext 3456, fax: 1-519-973-7073, e-mail: kwli@uwindsor.ca.
} 
reduce their costs by at least 30 percent. Otherwise, they will lose their market to Chinese-made products.

Lower labor cost is considered as a classical account for the China price. However, Engardio et al. (2004) challenge this interpretation by pointing out that direct labor only contributes less than 10 percent of total costs for sophisticated electronics. Nevertheless, Chinese-made products still hold a considerable competitive advantage. To investigate other possible factors, Bhattacharya et al. (2004) empirically cite lower capital-investment costs, lower domestic-sourcing costs, greater economies of scale, and government incentives. Wu et al. (2006) argue that lower labor costs by themselves are not necessarily transformed into lower product prices. Following the idea of industrial clusters (Porter, 1998), they propose that supply chain clusters play a central role in the transformation process, where most of the (vertically and/or horizontally) interrelated supply chain activities are geographically located nearby.

Porter (1998) claims that cluster members are entitled to utilize a "common glue" to realize extra economic values. The common glue takes the form of social networks (Porter 1998; Gordon and McCann 2000), integrated knowledge (Morosini 2004), input resources, and innovation incentive mechanisms, to name a few. In short, a cluster creates a common platform that facilitates its members to improve productivity.

If the common platform is provided and owned by an economic agent, it is reasonable to argue that the firms in a cluster are factually outsourcing to the agent and the nature of the cluster is simply an outcome of MCO.

From an economics perspective, the improvement of productivity via MCO can be interpreted in terms of both scope and scale economies: sharing and jointly utilization of inputs (Panzar and Willig1977, 1981; Bailey and Friedlaender 1982). However, as all functions are unlikely to increase or decrease proportionally, the concept of ray scale economies is not applicable in a strict sense. Instead, scope economies are a more appropriate vehicle as it does not require proportional change of inputs. Therefore, we will investigate how firms take advantage of scope economies to improve productivity and examine the impact of MCO on market prices.

Consider two competitors who seek potential outsourcing opportunities and a vendor that possesses a technology characterized by a parameter representing the degree of scope economies to perform outsourced functions. Following the same idea of Cachon and Harker (2002) and van Mieghem (1999), it is assumed that the vendor only provides outsourcing services and does not sell end-products to consumers directly. The vendor moves first to decide whether to offer an outsourcing service schedule to provide the contemplated outsourcing services. ${ }^{1}$ Then the two firms move simultaneously (or sequentially) to make their outsourcing decisions of the make-or-buy type. Finally the two firms compete in a final market. In the game theory terminology, a three-stage (or four-stage) game model has been built and the concept of subgame perfect equilibrium will be employed to examine the game.

Following the line of transaction cost theory (TCT) (Coase 1937; Williamson 1975; Cheung 1983; Grossman and Hart 1986; Hart and Moore 1990), researchers have focused on single-client (one vendor versus one client) outsourcing decisions modeled as make-or-buy problems, ${ }^{2}$ which are typically treated as a cost-benefit analysis. The trade-offs between in-house production and outsourcing may theoretically be treated as comparison of agency costs among different information structures (Sridhar and Balachandran 1997), of incentive effectiveness due to asset specificity (Chalos and Sung 1998), or of transaction costs based on two-sided informational asymmetry (Whang 1992). Osei-Bryson and Ngwenyama (2006) develop a system of practical methods and tools to quantify outsourcing risks and benefits and, then, to structure

\footnotetext{
${ }^{1}$ For simplification, we assume that the vendor's infrastructure is available for use and the setup cost is neglected.

${ }^{2}$ For a more detailed literature survey on outsourcing, see Debbern et al. (2004).
} 
inventive contracts. To this end, the single-client framework has been well explored.

However, very few formal studies address MCO even if it has been widely observed and conceptually analyzed by some authors. ${ }^{3}$ Cachon and Harker (2002), with a game model of two-firm full price competition under scale economies, prove that collectively outsourcing (resulting in a constant marginal cost) mitigates price competition and, then, makes both firms better off while in-house production with scale economies (resulting in a decreased average cost) raises price competition. They thus conclude that scale economies provide a strong motivation for outsourcing. Dube et al. (2007) examine a simpler version of Cachon and Harker (2002) where scale economies do not exist. They point out that the cost saving (the in-house production cost minus the outsourcing cost) drives firms to outsource.

Our model focuses on examining another motivation: the combination of high in-house production costs and the advantage of scope economies. We try to explain the occurrence of MCO and investigate its impact on market prices. This model is different from that in Cachon and Harker (2002) in three aspects: Firstly, firms here compete in the final market through quantity rather than price. ${ }^{4}$ Secondly, firms here attempt to proactively take advantage of scope economies via MCO while, in Cachon and Harker (2002), firms tend to avoid their in-house scale economies by outsourcing to a vendor at a constant wholesale price so as to mitigate price competition. Thirdly, MCO leads to lower equilibrium prices in the final market if the degree of scope economies is high enough, while in Cachon and Harker (2002), collective outsourcing drives equilibrium prices higher.

The rest of the paper is organized as follows. Section 2 presents a dynamic game model to describe the economic situations under consideration. Section 3 reports the equilibriums of the game. Section 4 is devoted to refining the equilibriums. Section 5 provides some comparative statics about the refined equilibrium. Section 6 furnishes additional discussions on relaxing the firms' risk-neutral assumption to be risk-averse, and symmetric cost assumption to be asymmetric. Some concluding remarks are provided in Section 7.

\section{The Model}

Consider two firms (denoted by $i$ and $j, i, j=1,2$ ), each of which has a similar but non-identical function and provides products or services to their consumers with (inverse) demand curves ${ }^{5}$

$$
p_{i}=1-q_{i}-r q_{j} \quad(i, j \in\{1,2\}, i \neq j)
$$

where $r \in[0,1]$ represents the degree of substitution between product (or service) $i$ and $j$, and $q_{i} \in[0,1], i=1,2$.

There exists an independent vendor with the capacity implementing these similar functions. Firm $i(i=1,2)$ either carries out such a function by itself or turns it over to the external vendor. These two alternatives are referred to as "make (M)" and "buy (B)", respectively.

Assume that firm $i(i=1,2)$ is risk-neutral and has a constant-return-to-scale technology with an expected unit cost $c_{i}$ and a variance $\sigma_{F i}^{2}$ to "make" its products or services. So the expected cost function is $C_{M_{i}}\left(q_{i}\right)=c_{i} q_{i}$ when $\mathrm{M}$ is chosen. To concentrate on exploring an alternative motivation of MCO (and

\footnotetext{
${ }^{3}$ See, for example, Sharma and Yetton (1996) and Gallivan and Oh (1999).

${ }^{4}$ Competing on quantity or price is generally regarded as two alternative rules for market competitions. It is generally accepted that the latter is fiercer than the former. We take the milder one because it entitles us to explore more details about how MCO takes advantage of scope economies and leads to lower market prices.

${ }^{5}$ Although a general linear demand function should take the form of $p_{i}=a-b q_{i}-r q_{j}$, our assumption of demand functions is equivalent to the general form if we appropriately manipulate the units used to measure currency, product $i$ and product $j$.
} 
then the formation of supply chain cities) in terms of scope economies, we assume the two firms are symmetric in the sense that both the expectation and variance of their marginal costs are the same (i.e. $c_{1}=c_{2}=c<1$ and $\sigma_{F 1}^{2}=\sigma_{F 2}^{2}=\sigma^{2}$ ) if both choose M. ${ }^{6}$ If firm $i$ decides to "buy", it will sign a contract with the vendor specifying a wholesale price, $\alpha_{i}$, for the outsourced products or services. Thus its cost function is $C_{i}\left(q_{i}\right)=\alpha_{i} q_{i}$.

The vendor is assumed to be risk-averse with a constant Arrow-Pratt measure of absolute risk aversion $\rho>0$, and owns a technology with the following cost function ${ }^{7}$

$$
C_{v}\left(q_{1}, q_{2}\right)= \begin{cases}0 & q_{1}=q_{2}=0 \\ c_{v}^{1} q_{1} & q_{1}>0, q_{2}=0 \\ c_{v}^{2} q_{2} & q_{2}>0, q_{1}=0 \\ k\left(c_{v}^{1} q_{1}+c_{v}^{2} q_{2}\right) & q_{2}>0, q_{1}>0\end{cases}
$$

where $k \in(0,1]$ captures the nature of scope economies: it is cheaper to produce multiple products jointly than separately and the smaller the $k$ is, the stronger the scope economies, and $c_{v}^{i}(i=1,2)$ is the vendor's random unit cost of separately serving firm $i$. To study outsourcing decision with the problem of managerial incentives, Chalos and Sung (1998) take the same assumption that the outsourcer is risk-neutral while the vendor is risk-averse. An empirical interpretation of this risk-neutral firms and risk-averse vendor assumption is that outsourcing firms tend to be larger corporations with diversified business while a vendor is more likely to be a specialized firm with limited diversification. Nevertheless, Section 6 relaxes the risk-neutrality assumption to allow the firms to be risk-averse so that the model may accommodate the case that firms are not so-well-diversified and, hence, share the risk-averse characteristic with the vendor.

In the base model, it is further assumed that $c_{v}^{1}$ and $c_{v}^{2}$ are independent with $E c_{v}^{1}=c_{1}, E c_{v}^{2}=c_{2}$ and $\operatorname{var}\left(c_{v}^{1}\right)=\operatorname{var}\left(c_{v}^{2}\right)=\sigma^{2}$. In so doing, different expected costs between $E c_{v}^{i}$ and $c_{i}(i=1,2)$ are excluded for the sake of analytical tractability. ${ }^{8}$ Furthermore, the cost function (1) implies that the vendor's technology yields a constant return to scale (i.e., for any given $c_{v}^{1}, c_{v}^{2}$ and $k, C_{v}\left(t q_{1}, t q_{2}\right)=t C_{v}\left(q_{1}, q_{2}\right)$ holds for all $t \geq 0$ ), thereby excluding any potential impact of scale economies (and diseconomies). Thus, by excluding cost differences between the vendor and firm $i$ (when separately serving) and scale economies (diseconomies), this research focuses on the impact of scope economies (captured by $k$ ) on the firms' outsourcing decisions.

Since the vender's production quantity is contingent upon firm $i$ 's and $j$ 's make-or-buy decisions, four cases are to be examined: Case 1. $q_{1}=q_{2}=0$; Case 2. $q_{1}>0, q_{2}=0$; Case 3. $q_{2}>0, q_{1}=0$; and Case 4. $q_{2}>0, q_{1}>0$. Case 1 is trivial: the vendor's optimal production is simply not to produce at all. We ignore the trivial case and analyze the other three cases more carefully below.

With the concept of certainty equivalence, the vendor's profit-maximizing decision in case 2 can be

\footnotetext{
${ }^{6}$ If $c \geq 1$, it is not profitable for the firms to provide any product (or service) to consumers.

${ }^{7}$ Here, $q_{i}(i=1,2$ ) should be understood as the quantity ordered by outsourcer $i$ (i. e. the Cournot equilibrium quantity).

${ }^{8}$ First, this technical assumption $E c_{v}^{i}=c_{i}$ (similar to the treatment in Cachon and Harker (2002)) implies a setting where the vendor has no technological advantage when he/she separately serves firm $i$ (i.e. $q_{i}>0, q_{j}=0$ ). Second, with the symmetric cost assumption, $E c_{v}^{1}=c_{1}=c=c_{2}=E c_{v}^{2}$, which remains valid up until Section 5 but will be relaxed in Section 6 , the cost difference between the two firms (and then its impact on the market equilibrium) is excluded. Third, $\operatorname{var}\left(c_{v}^{i}\right)=\sigma^{2}\left(=\sigma_{F i}^{2}\right)$ implies the vendor operates in the same exogenous stochastic environment in terms of volatility as firm $i$ (this assumption, once again, remains valid up until Section 5 but will be relaxed in Section 6). And finally, the independence assumption excludes the interaction of the exogenous stochastic impacts of the vendor's environment on the costs of serving both firms when they co-source. The overall purpose of these assumptions is to isolate scope economies from other factors and focus on the impact of scope economies on firms' outsourcing decisions.
} 
written as

$$
\max _{q_{1}>0}\left\{\alpha_{1} q_{1}-c q_{1}-\frac{1}{2} \rho \sigma^{2} q_{1}^{2}\right\}
$$

The vendor's “supply” curve (the first-order condition) in case 2 is

$$
\alpha_{1}=c+\rho \sigma^{2} q_{1}
$$

Similarly, the "supply" curve in case 3 is

$$
\alpha_{2}=c+\rho \sigma^{2} q_{2}
$$

(2) and (3) imply that when firm $j$ does not buy from the vendor, the purchasing cost of firm $i$ is

$$
C_{B_{i}, M_{j}}\left(q_{i}\right)=c q_{i}+\rho \sigma^{2} q_{i}^{2} \quad(i=1,2)
$$

In case 4, the "supply" curves are

$$
\alpha_{i}=k c+\rho \sigma^{2} k^{2} q_{i} \quad(i=1,2)
$$

In (4), the first term corresponds to the vendor's expected production cost while the second represents risk cost. Comparing (4) with firm $i$ 's in-house production cost function $C_{M_{i}}\left(q_{i}\right)=c q_{i}$, the difference is $\Delta C_{B_{i}, M_{j}}\left(q_{i}\right)=\rho \sigma^{2} q_{i}^{2}$ which is exactly the vendor's risk cost, where firm $i$ chooses $\mathrm{B}$ and $j$ selects $\mathrm{M}$.

In the presence of scope economies when both firms outsource to the vendor, the difference is

$$
\Delta C_{B_{i}, B_{j}}\left(q_{i}\right)=(k-1) c q_{i}+\rho \sigma^{2} k^{2} q_{i}^{2}
$$

where the first term represents the production cost savings while the second term stands for the additional risk cost when both firms decide to outsource.

Given firm $i$ 's market decision ( $q_{i}$ is fixed), $M$ has a cost advantage if $\Delta C_{B_{i}, d_{j}}\left(q_{i}\right) \geq 0 \quad(d \in\{M, B\})$, otherwise $\mathrm{B}$ is more cost-effective than $\mathrm{M}$. For a given $q_{i}$, the cost advantage/disadvantage essentially dictates the make-or-buy decision. This result is consistent with a well-known proposition that the difference of transaction costs determines the boundary of a firm (Coase 1937, Williamson 1975).

Next, we will relax this fixed market decision assumption and, then, introduce scope economies into a three-stage game model to investigate the make-or-buy decision under the condition of quantity competition in the final consumer market. The sequence of events is as follows. In stage 1 , the vendor decides whether to offer the outsourcing service according to the cost function in (1). Then, firms $i$ and $j$ simultaneously make their make-or-buy decisions in stage 2, designated as the make-or-buy (MOB) game. ${ }^{9}$ Finally, firms $i$ and $j$ compete against each other in the consumer market, named below as the market game. The whole decision process is referred to as $G_{O}$.

\section{The Equilibriums}

Now the concept of sub-game perfect equilibrium is applied to analyze the three-stage game model by backward induction. First, we solve the market game to derive the payoff matrix for the MOB game. Clearly, the strategy profiles of the $\mathrm{MOB}$ game are $\{M, M\},\{M, B\},\{B, M\}$ and $\{B, B\}$ where the first and second element in a strategy profile refer to firm 1's and 2's strategy, respectively. For $\{\mathrm{M}, \mathrm{M}\}$, firm $i$ 's ( $i=1,2$ ) market decision can be described as

$$
\pi_{i}(M, M)=\max _{q_{i}>0}\left\{\left(1-q_{i}-r q_{j}\right) q_{i}-c q_{i}\right\}
$$

\footnotetext{
${ }^{9}$ In section 4, the two firms will make their make-or-buy decisions sequentially rather than simultaneously.
} 
Firm $i$ 's reaction function is

$$
q_{i}(M, M)=\frac{1-c-r q_{j}}{2}(i \neq j)
$$

Then the market equilibrium quantity and payoff are

$$
q_{i}^{*}(M, M)=\frac{1-c}{2+r}, \quad \pi_{i}^{*}(M, M)=\left(\frac{1-c}{2+r}\right)^{2} \quad(i=1,2)
$$

Similarly, from Eq. (5), the market equilibrium quantity and payoff for $\{B, B\}$ are obtained as

$$
q_{i}^{*}(B, B)=\frac{1-k c}{2\left(1+\rho \sigma^{2} k^{2}\right)+r}, \quad \pi_{i}^{*}(B, B)=\left(1+\rho \sigma^{2} k^{2}\right)\left[\frac{1-k c}{2\left(1+\rho \sigma^{2} k^{2}\right)+r}\right]^{2} \quad(i=1,2)
$$

With (2) and (3), the market equilibrium for $\{\mathrm{B}, \mathrm{M}\}$ can be derived as

$$
q_{1}^{*}(B, M)=\frac{(2-r)(1-c)}{4\left(1+\rho \sigma^{2}\right)-r^{2}}, \quad \pi_{1}^{*}(B, M)=\left(1+\rho \sigma^{2}\right)\left[\frac{(2-r)(1-c)}{4\left(1+\rho \sigma^{2}\right)-r^{2}}\right]^{2}
$$

and

$$
q_{2}^{*}(B, M)=\frac{\left(2\left(1+\rho \sigma^{2}\right)-r\right)(1-c)}{4\left(1+\rho \sigma^{2}\right)-r^{2}}, \quad \pi_{2}^{*}(B, M)=\left[\frac{\left(2\left(1+\rho \sigma^{2}\right)-r\right)(1-c)}{4\left(1+\rho \sigma^{2}\right)-r^{2}}\right]^{2}
$$

By symmetry, the market equilibrium for $\{\mathrm{M}, \mathrm{B}\}$ can be similarly obtained as

$$
q_{1}^{*}(M, B)=\frac{\left(2\left(1+\rho \sigma^{2}\right)-r\right)(1-c)}{4\left(1+\rho \sigma^{2}\right)-r^{2}}, \pi_{1}^{*}(M, B)=\left[\frac{\left(2\left(1+\rho \sigma^{2}\right)-r\right)(1-c)}{4\left(1+\rho \sigma^{2}\right)-r^{2}}\right]^{2}
$$

and

$$
q_{2}^{*}(M, B)=\frac{(2-r)(1-c)}{4\left(1+\rho \sigma^{2}\right)-r^{2}}, \quad \pi_{2}^{*}(M, B)=\left(1+\rho \sigma^{2}\right)\left[\frac{(2-r)(1-c)}{4\left(1+\rho \sigma^{2}\right)-r^{2}}\right]^{2}
$$

Thus, the MOB game can be described as the following $2 \times 2$ matrix form.

Table 1 The Normal-Form Make-or-Buy Game

\begin{tabular}{lcccc}
\hline & \multicolumn{4}{c}{ Firm 2 } \\
\cline { 2 - 4 } Firm 1 & M & $\pi_{1}^{*}(M, M), \pi_{2}^{*}(M, M)$ & $\pi_{1}^{*}(M, B), \pi_{2}^{*}(M, B)$ \\
& & & & \\
& B & $\pi_{1}^{*}(B, M), \pi_{2}^{*}(B, M)$ & $\pi_{1}^{*}(B, B), \pi_{2}^{*}(B, B)$ \\
\hline
\end{tabular}

Lemma 1: Given the vendor's outsourcing service schedule characterized by (2), (3), and (5), the strategy $\{M, M\}$ is always a Nash equilibrium, but neither $\{B, M\}$ nor $\{M, B\}$ can be a Nash equilibrium.

Proof: Given that both firms choose $\mathrm{M}$, as $1+\rho \sigma^{2}>1$, we have

$$
\left(\frac{\pi_{1}^{*}(M, M)}{\pi_{1}^{*}(B, M)}\right)^{1 / 2}=\frac{4 \sqrt{\left(1+\rho \sigma^{2}\right)}-r^{2} / \sqrt{\left(1+\rho \sigma^{2}\right)}}{4-r^{2}}>1
$$

Hence, $\pi_{1}^{*}(M, M)>\pi_{1}^{*}(B, M)$. By symmetry, $\pi_{2}^{*}(M, M)>\pi_{2}^{*}(M, B)$. As none of the two firms has 
incentive to deviate from their current strategy, $\{\mathrm{M}, \mathrm{M}\}$ is a Nash equilibrium. The second part is straightforward. Lemma 1 is thus proved.

Lemma 1 shows that neither firm has the incentive to outsource if he/she anticipates that his/her opponent does not outsource. The reason is that the outsourcing firm has to bear an extra cost $\Delta C_{B_{i}, M_{j}}\left(q_{i}\right)=\rho \sigma^{2} q_{i}^{2} \quad$ (relative to in-house production) caused by the risk-averse vendor's optimal supply behavior (see (2) or (3)). In this case, any sole outsource increases the cost of the outsourcing firm, resulting in a competitive disadvantage in the final (differentiable) product market under quantity competition. This argument can also be applied to the situation where the firms are risk averse (see Section 6). Next, Proposition 1 below reveals that it is an important concern whether scope economies are realized via MCO when firms make their make-or-buy decisions. The reason is that $\Delta C_{B_{i}, B_{j}}\left(q_{i}\right)=(k-1) c q_{i}+\rho \sigma^{2} k^{2} q_{i}^{2}$ increases in $k$, or alternatively, $\Delta C_{B_{i}, B_{j}}$ deceases as the degree of scope economies increases. Eventually, when the degree of scope economies exceeds a threshold, (B, B) will arise as another Nash equilibrium.

Let

$$
c_{0}=\frac{2 r \rho \sigma^{2}}{4\left(1+\rho \sigma^{2}\right)-r^{2}+2 r \rho \sigma^{2}}>0
$$

Proposition 1: Given the vendor's outsourcing service schedule described by (2), (3) and (5), if $c>c_{0}$, there exists a critical $k_{0} \in(0,1)$ such that $\{B, B\}$ is a Nash equilibrium for any $k<k_{0}$.

Proof: Define $G:[0,1] \rightarrow \mathrm{R}$ as $G(k)=\pi_{1}^{*}(B, B)-\pi_{1}^{*}(M, B)$, then

$$
G(k)=\left[\frac{1-k c}{2 \sqrt{1+\rho \sigma^{2} k^{2}}+r / \sqrt{1+\rho \sigma^{2} k^{2}}}\right]^{2}-\left[\frac{\left(2\left(1+\rho \sigma^{2}\right)-r\right)(1-c)}{4\left(1+\rho \sigma^{2}\right)-r^{2}}\right]^{2}
$$

For any given $r \in[0,1]$, let $x=\sqrt{1+\rho \sigma^{2} k^{2}}$ and $f(x)=2 x+r / x$ It is obvious that $f(x)$ is strictly increasing on $[1, \infty)$. Then for all $k \in(0,1)$, we have

$$
G^{\prime}(k)=2\left[\frac{1-k c}{2 \sqrt{1+\rho \sigma^{2} k^{2}}+r / \sqrt{1+\rho \sigma^{2} k^{2}}}\right] \times\left[\frac{-c}{f\left(\sqrt{1+\rho \sigma^{2} k^{2}}\right)}-\frac{\rho \sigma^{2} k(1-k c) f^{\prime}\left(\sqrt{1+\rho \sigma^{2} k^{2}}\right)}{\left[f\left(\sqrt{1+\rho \sigma^{2} k^{2}}\right)\right]^{2} \sqrt{1+\rho \sigma^{2} k^{2}}}\right]<0
$$

Furthermore, since $\rho>0, \sigma^{2}>0$ and $r \in[0,1]$, it is easy to check

$$
G(0)=\left[\frac{1}{2+r}\right]^{2}-\left[\frac{\left(2\left(1+\rho \sigma^{2}\right)-r\right)(1-c)}{4\left(1+\rho \sigma^{2}\right)-r^{2}}\right]^{2}>0
$$

for all $c>c_{0}$.

Next, we shall prove that $G(1)<0$. First let $y=\sqrt{1+\rho \sigma^{2}}>1$, then $4\left(y^{4}-y^{3}\right)>0>r^{2}(1-y)$, i.e. $4 y^{4}-r^{2}>y\left(4 y^{2}-r^{2}\right)$, thus

$$
0<\frac{y}{2 y^{2}+r}<\frac{2 y^{2}-r}{4 y^{2}-r^{2}}
$$

By plugging $y=\sqrt{1+\rho \sigma^{2}}>1$ back into $G(1)$, we have 


$$
G(1)=\left[\left(\frac{\sqrt{1+\rho \sigma^{2}}}{2\left(1+\rho \sigma^{2}\right)+r}\right)^{2}-\left(\frac{2\left(1+\rho \sigma^{2}\right)-r}{4\left(1+\rho \sigma^{2}\right)-r^{2}}\right)^{2}\right](1-c)^{2}<0
$$

Clearly, $G(\cdot)$ is continuous on $(0,1]$. Then if $c>c_{0}, G(0)>0, G(1)<0$ and $G^{\prime}(\cdot)<0$ imply that there exists a critical $k_{0} \in(0,1)$ such that $G\left(k_{0}\right)=0, G(k)>0$ for all $k<k_{0}$, and $G(k)<0$ for all $k>k_{0}$. By the symmetry of the MOB game and the definition of $G(\cdot),\{\mathrm{B}, \mathrm{B}\}$ is a Nash equilibrium. Proposition 1 is then proved.

Corollary 1: Given the vendor's outsourcing service schedule described by (2), (3) and (5), $\{M, M\}$ is the one and only one Nash equilibrium if either $c \leq c_{0}$ or $k>k_{0}$.

Proof: By the definition of $G(\cdot)$, if $k>k_{0}, G(k)<0$ holds for all $c \in(0,1)$. Then we have $\pi_{1}^{*}(B, B)<\pi_{1}^{*}(M, B)$. Thus $\{\mathrm{B}, \mathrm{B}\}$ is not a Nash equilibrium in this case. Similarly, if $c<c_{0},\{\mathrm{~B}, \mathrm{~B}\}$ is not a Nash equilibrium either for all $k \in(0,1]$.

For the trivial case of $c=c_{0}$, we have $G(0)=0$, then $\{\mathrm{B}, \mathrm{B}\}$ might also be a Nash equilibrium. However, by the strict monotonicity of $G(\cdot)$ with respect to $k$ and the definition of $k_{0}\left(G\left(k_{0}\right)=0\right)$, we have $k_{0}=0$. This is impossible as there does not exist such a level of scope economies (corresponding to a zero production cost for the vendor). Therefore, $\{\mathrm{B}, \mathrm{B}\}$ cannot be a Nash equilibrium. Furthermore, Lemma 1 establishes that $\{M, M\}$ is a Nash equilibrium regardless of the values of $c$ and $k$ and that neither $\{B$, $\mathrm{M}\}$ nor $\{\mathrm{M}, \mathrm{B}\}$ is a Nash equilibrium. Corollary 1 is thus proved.

Corollary 1 clearly demonstrates that it is impossible for MCO to emerge when scope economies are not strong enough $\left(k>k_{0}\right)$ or the firms' in-house production cost is not sufficiently high $\left(c \leq c_{0}\right)$. As both the cost and scope economies reach sufficiently high levels ( $c>c_{0}$ and $k<k_{0}$ ), firms tend to outsource to an outside vendor instead of producing in-house. This is consistent with our intuition. Under quantity competition in the final market, firms seek to reduce their cost to achieve a higher profit when the in-house production cost is high $\left(c>c_{0}\right)$. In this case, MCO serves as a means to attaining cost-savings by exploiting the advantage of scope economies. This result indicates that scope economies furnish a reasonable explanation for the occurrence of MCO (and then the formation of supply chain cities). Cachon and Harker (2002) suggest that economies of scale provide a strong motivation for collectively outsourcing (MCO) under price competition. Here we explore the potential of scope economies to be an alternative motivation. However, when in-house production costs are sufficiently low, regardless of the level of scope economies, both firms will choose M, thereby preventing the occurrence of MCO. Therefore, in-house production cost is an inalienable factor when we examine the emergence of MCO.

Remark 1: Two points should be clarified here. Firstly, along with Corollary 1, Proposition 1 implies that, given certain parameters, there exists a functional relationship (determined by $G(k)=0)$ between the critical level of scope economies $\left(k_{0}\right)$ and $c$ if $c>c_{0}$ such that MCO may occur. However, the property of this relationship is yet to be explored, and will be addressed in Section 5 when comparative static properties are examined. Secondly, because the MOB game has two Nash equilibriums, $\{M, M\}$ and $\{B, B\}$, we can only conclude that it is possible that MCO occurs when both firms make their choices simultaneously. In Section 4, we will slightly modify the MOB game by allowing sequential moves by the two firms to refine the Nash equilibriums.

Now we turn to the vendor's decision in stage 1. The vendor has two alternatives: Offer (O) and Not Offer (N) the outsourcing service schedule as characterized by (2), (3), and (5). If it chooses $\mathrm{N}$, its payoff is simply $\pi_{v}(N)=0$. Otherwise, if the vendor offers the service, its payoff is contingent upon which 
equilibrium eventually emerges: if $\{\mathrm{M}, \mathrm{M}\}$ is realized, its payoff is also $\pi_{v}(O)=0$; but an equilibrium $\{\mathrm{B}$, B implies a payoff of $\pi_{v}(O)=\rho \sigma^{2} k^{2}\left[\left(q_{1}^{*}(B, B)\right)^{2}+\left(q_{2}^{*}(B, B)\right)^{2}\right] / 2$. In any case, $\pi_{v}(O) \geq 0=\pi_{v}(N)$. Therefore, the vendor will choose $\mathrm{O}$.

To summarize, $G_{O}$ may have two subgame perfect equilibriums with the following equilibrium paths:

$$
\begin{aligned}
& \mathrm{E} 1: \mathrm{O} \rightarrow\{\mathrm{M}, \mathrm{M}\} \rightarrow\left\{q_{1}^{*}(M, M), q_{2}^{*}(M, M)\right\} \\
& \mathrm{E} 2: \mathrm{O} \rightarrow\{\mathrm{B}, \mathrm{B}\} \rightarrow\left\{q_{1}^{*}(B, B), q_{2}^{*}(B, B)\right\}
\end{aligned}
$$

where E1 is always a subgame perfect equilibrium and E2 becomes an equilibrium only if the firms' in-house production cost exceeds a certain threshold $\left(c>c_{0}\right)$ and the scope economies reach a certain level $\left(k<k_{0}\right)$.

\section{Refinement of the Equilibriums}

Analytical results in Section 3 indicate that $c>c_{0}$ and $k<k_{0}$ are necessary conditions for $\{\mathrm{B}, \mathrm{B}\}$ becoming a subgame perfect equilibriums of $G_{O}$. However, as there exists another equilibrium $\{\mathrm{M}, \mathrm{M}\}$, it remains uncertain which one will come up as the final solution. On the other hand, the existence of these two equilibriums is related to the assumption of simultaneous moves. But in reality, firms seldom make their outsourcing decision simultaneously. In order to make our model more consistent with general business practice, we will refine the equilibriums of this subgame by assuming the two firms making sequential moves. ${ }^{10}$

Due to the symmetry of the MOB game, without loss of generality, we assume that firm 1 moves first and firm 2 follows. The modified MOB game can be depicted in Figure 1. The corresponding full game is named the modified outsourcing game and denoted by $G_{O}^{\prime}$

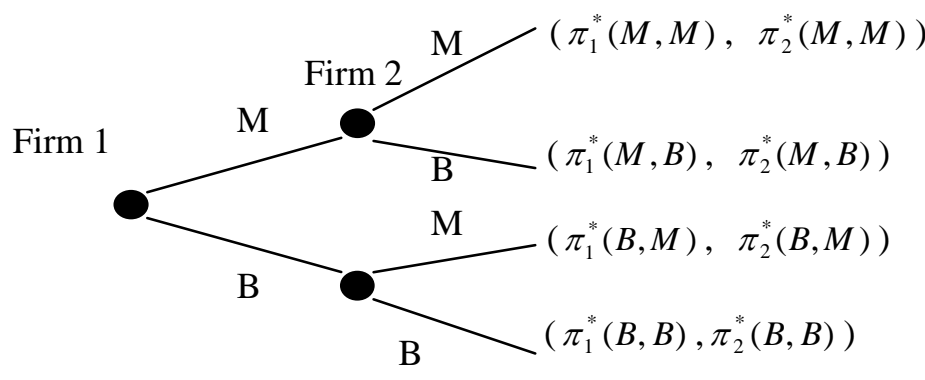

Figure 1 The Extensive-Form Make-or-Buy Game

Proposition 2: Given the vendor's outsourcing service schedule described by (2), (3) and (5), when $c>c_{0}$, $\{B, B\}$ is a unique subgame perfect equilibrium of the modified MOB game in Figure 1 for all $k<k_{0}$.

Proof: Lemma 1 implies that firm 2 will choose $M$ if firm 1 chooses $M$ first. In this case, firm 1's eventual payoff is $\pi_{1}^{*}(M, M)$ when it selects $\mathrm{M}$.

Next, Define $H:[0,1] \rightarrow \mathrm{R}$ as $H(k)=\pi_{1}^{*}(B, B)-\pi_{1}^{*}(M, M)$. It is easy to check that, for any $k,(6)$ and (9) imply

\footnotetext{
${ }^{10}$ Here the concept of subgame perfect equilibrium is employed to refine the Nash equilibriums obtained in Section 3. In game theory, there are other equilibrium concepts for refinement such as Bayesian perfect equilibrium, sequential equilibrium, perfect equilibrium, and proper equilibrium. However, these are not appropriate for our case as there is no information asymmetry in our model. Therefore, it is unnecessary to consider the equilibrium of beliefs that is the main concern of these concepts. For detailed explorations of Nash equilibrium refinement, see Vega-Redondo (2003, Ch. 4).
} 


$$
\sqrt{\frac{\pi_{1}^{*}(M, M)}{\pi_{1}^{*}(M, B)}}=\frac{4\left(1+\rho \sigma^{2}\right)-r^{2}}{4\left(1+\rho \sigma^{2}\right)-r^{2}+2 r \rho \sigma^{2}}<1
$$

Then we have

$$
\begin{aligned}
G(k)-H(k) & =\left[\pi_{2}^{*}(B, B)-\pi_{2}^{*}(B, M)\right]-\left[\pi_{1}^{*}(B, B)-\pi_{1}^{*}(M, M)\right] \\
& =\left[\pi_{1}^{*}(B, B)-\pi_{1}^{*}(M, B)\right]-\left[\pi_{1}^{*}(B, B)-\pi_{1}^{*}(M, M)\right] \\
& =\pi_{1}^{*}(M, M)-\pi_{1}^{*}(M, B)<0
\end{aligned}
$$

Therefore, $H(k)>G(k)>0$ for any $k<k_{0}$, since $G(k)>0$ for all $k<k_{0}$.

Note that $\pi_{2}^{*}(B, B)>\pi_{2}^{*}(B, M)$ for all $k<k_{0}$. So, firm 2 will choose B if firm 1 chooses B first. In addition, for all $k<k_{0}$, we have $\pi_{1}^{*}(B, B)>\pi_{1}^{*}(M, M)$. This means that firm 1 is motivated to choose $\mathrm{B}$. This proves Proposition 2.

Remark 2: Proposition 2 shows that, after the Nash equilibriums are refined, the strategy profile $\{\mathrm{B}, \mathrm{B}\}$ becomes the unique subgame perfect equilibrium as long as scope economies are strong enough $\left(k<k_{0}\right)$ and the in-house production cost is high enough $c>c_{0}$. In the terminology of game theory, we rule out the incredible threat that firm 1 chooses $M$ and, thus $\{M, M\}$ (and then $E 1$ ) is no longer a credible equilibrium. Note that the proof does not place any restriction on the values of other parameters such as $r, \rho$, and $\sigma^{2}$. The implication is that there always exists a critical degree of scope economies such that both firms are motivated to outsource to an outside vendor for any given profile of $r, \rho$, and $\sigma^{2}$ as long as $c>c_{0}$. The relevance of this result to business practice is that, if firms make their outsourcing decisions sequentially rather than simultaneously and their in-house production cost and scope economies both reach sufficiently high levels, their similar (operational) functions will be outsourced to and performed by an outside vendor regardless of the exogenous business environment. Therefore, this analysis provides a reasonable account for the emergence of supply chain cities/clusters via MCO.

\section{The Comparative Statics}

Now we investigate how $k_{0}$ changes as the parameter values $\left(r, c \in\left(c_{0}, 1\right), \rho\right.$ and $\left.\sigma^{2}\right)$ vary and the impact of collective outsourcing on market prices.

Proposition 3: Given the values of other parameters, $k_{0}$ increases in $c$, but decreases in $\rho, \sigma^{2}$ and $r$.

Proof: Define $W:[0,1] \rightarrow \mathrm{R}$ as

$$
W(k)=\frac{1-k c}{2 \sqrt{1+\rho \sigma^{2} k^{2}}+r / \sqrt{1+\rho \sigma^{2} k^{2}}}-\frac{\left(2\left(1+\rho \sigma^{2}\right)-r\right)(1-c)}{4\left(1+\rho \sigma^{2}\right)-r^{2}}
$$

Clearly, $G\left(k_{0}\right)=0$ is equivalent to $W\left(k_{0}\right)=0$. Now related partial derivatives of $W$ are determined as follows.

$$
\frac{\partial W}{\partial k}=\frac{-c}{f\left(\sqrt{1+\rho \sigma^{2} k^{2}}\right)}-\frac{\rho \sigma^{2} k(1-k c) f^{\prime}\left(\sqrt{1+\rho \sigma^{2} k^{2}}\right)}{\left[f\left(\sqrt{1+\rho \sigma^{2} k^{2}}\right)\right]^{2} \sqrt{1+\rho \sigma^{2} k^{2}}}<0
$$




$$
\left.\begin{array}{c}
\frac{\partial W}{\partial c}=\frac{-k}{f\left(\sqrt{1+\rho \sigma^{2} k^{2}}\right)}+\frac{2\left(1+\rho \sigma^{2}\right)-r}{4\left(1+\rho \sigma^{2}\right)-r^{2}} \geq \frac{-1}{f\left(\sqrt{1+\rho \sigma^{2} k^{2}}\right)}+\frac{2\left(1+\rho \sigma^{2}\right)-r}{4\left(1+\rho \sigma^{2}\right)-r^{2}} \\
>\frac{-1}{2+r}+\frac{2\left(1+\rho \sigma^{2}\right)-r}{4\left(1+\rho \sigma^{2}\right)-r^{2}}=\frac{2 r \rho \sigma^{2}}{\left.4\left(1+\rho \sigma^{2}\right)-r^{2}\right](2+r)}>0 \\
\frac{\partial W}{\partial \rho}=-\frac{\sigma^{2} k^{2}(1-k c) f^{\prime}\left(\sqrt{1+\rho \sigma^{2} k^{2}}\right)}{\left[f\left(\sqrt{1+\rho \sigma^{2} k^{2}}\right)\right]^{2} \sqrt{1+\rho \sigma^{2} k^{2}}}-\frac{2 r \sigma^{2}(2-r)(1-c)}{\left[4\left(1+\rho \sigma^{2}\right)-r^{2}\right]^{2}}<0 \\
\frac{\partial W}{\partial r}=\frac{\frac{\partial W}{\partial \sigma^{2}}=-\frac{\rho k^{2}(1-k c) f^{\prime}\left(\sqrt{1+\rho \sigma^{2} k^{2}}\right)}{\left[f\left(\sqrt{1+\rho \sigma^{2} k^{2}}\right)\right]^{2} \sqrt{1+\rho \sigma^{2} k^{2}}}-\frac{4 r \rho(2-r)(1-c)}{\left[4\left(1+\rho \sigma^{2}\right)-r^{2}\right]^{2}}<0}{\sqrt{1+\rho \sigma^{2} k^{2}}\left[f\left(\sqrt{1+\rho \sigma^{2} k^{2}}\right)\right]^{2}+\frac{\left(4\left(1+\rho \sigma^{2}\right)-4 r\left(1+\rho \sigma^{2}\right)+r^{2}\right)(1-c)}{\left[4\left(1+\rho \sigma^{2}\right)-r^{2}\right]^{2}}} \\
=\frac{1-c}{4\left(1+\rho \sigma^{2}\right)-r^{2}}\left[\frac{-\left(2\left(1+\rho \sigma^{2}\right)-r\right)}{\sqrt{1+\rho \sigma^{2} k^{2}} f\left(\sqrt{1+\rho \sigma^{2} k^{2}}\right)}+\frac{4\left(1+\rho \sigma^{2}\right)-4 r\left(1+\rho \sigma^{2}\right)+r^{2}}{4\left(1+\rho \sigma^{2}\right)-r^{2}}\right] \\
=\frac{1-c}{4\left(1+\rho \sigma^{2}\right)-r^{2}}\left[\frac{-\left(2\left(1+\rho \sigma^{2}\right)-r\right)}{2\left(1+\rho \sigma^{2} k^{2}\right)+r}+\frac{4\left(1+\rho \sigma^{2}\right)-4 r\left(1+\rho \sigma^{2}\right)+r^{2}}{4\left(1+\rho \sigma^{2}\right)-r^{2}}\right] \\
\leq \frac{1-c}{\left.4\left(1+\rho \sigma^{2}\right)-r^{2}\right]^{2}} \times \frac{-8 r \rho \sigma^{2}\left(1+\rho \sigma^{2}\right)}{2\left(1+\rho \sigma^{2}\right)+r}<0
\end{array}\right]
$$

where the second equality in $\partial W / \partial r$ is due to the critical condition $W(k)=0$, the first inequalities in $\partial W / \partial c$ and in $\partial W / \partial r$ are derived from the assumption of $k \in(0,1]$, and the second inequality in $\partial W / \partial c$ is obtained according to the fact that $f(x)$ is strictly increasing in $x$ for all $x \geq 1$ and the definition of $f(x)$ is given in the proof of Proposition 1 in Section 3.

Then we have ${ }^{11}$

$$
\frac{\mathrm{d} k_{0}}{\mathrm{~d} c}>0, \frac{\mathrm{d} k_{0}}{\mathrm{~d} \rho}<0, \frac{\mathrm{d} k_{0}}{\mathrm{~d} \sigma^{2}}<0, \frac{\mathrm{d} k_{0}}{\mathrm{~d} r}<0
$$

Proposition 3 is therefore proved.

Remark 3: Proposition 3 establishes that the critical value $k_{0}$ of scope economies for MCO to emerge increases as in-house production cost $c$ increases. Note that the higher the value of $k_{0}$, the lower the degree of scope economies. Hence, this result implies that a higher in-house production cost requires a lower critical degree of scope economies to foster the collective outsourcing result. Specifically, under the sequential-move assumption (a more reasonable description of real-world business settings), E2 becomes the unique subgame perfect equilibrium if $k<k_{0}$ and $c>c_{0}$. This uniqueness provides a precise prediction of MCO. It should be stressed that both conditions, $k<k_{0}$ and $c>c_{0}$, are required for E2 to be the unique subgame perfect equilibrium. For instance, if $c \leq c_{0}$, Corollary 1 in Section 3 proves that E2 is not an equilibrium and, hence, cannot be a subgame perfect equilibrium here. Therefore, scope economies alone can not explain the occurrence of MCO. On the other hand, if there do not exist scope economies (i.e., $k=1$ ), $\Delta C_{B_{i}, B_{j}}\left(q_{i}\right)=\Delta C_{B_{i}, M_{j}}\left(q_{i}\right)=\rho \sigma^{2} q_{i}^{2}>0$. This means that for firm $i$, in-house production is always better than

\footnotetext{
${ }^{11}$ It is assumed here that the changes are examined one at a time. For instance, when we investigate the relationship between $k_{0}$ and $c$, other parameter values are fixed and remain constant.
} 
outsourcing regardless of its opponent's strategy. Then E2 would not be an equilibrium, either. These arguments demonstrate that the combination of a high in-house production cost $\left(c>c_{0}\right)$ and a high degree of scope economies $\left(k<k_{0}\right)$ motivates firms to collectively outsource to an outside vendor. Consequently, this combination should be recommended as an alternative motivation for the occurrence of MCO, in addition to Cachon and Harker's (2002) claim that scale economies is a motivation for MCO. However, the motivation differs. The model here suggests the firms to exploit the advantage of scope economies, but Cachon and Harker (2002) indicate that the firms give up their in-house scale economies by collectively outsourcing in exchange for a constant wholesale price, thereby mitigating price competition in the final market and making them both better-off. Furthermore, this result furnishes a reasonable account for Porter's "common glue" in industrial clusters (Porter, 1998) and, then, for the recently-observed phenomenon of supply chain cities (Kahn, 2004).

Remark 4: Proposition 3 also establishes the comparative statics of $k_{0}$ with regard to $\rho, \sigma^{2}$, and $r$, respectively. These results are consistent with our intuition: other values remain constant, the higher the aversion of risk (the uncertainty, or the magnitude of substitution) is, the higher the critical degree of scope economies is needed for achieving collective outsourcing.

Proposition 4: $p_{i}^{*}(B, B)(i=1,2)$ decreases as the degree of scope economies increases. Furthermore, there exists a $k_{m} \in(0,1)$ such that $p_{i}^{*}(B, B) \leq p_{i}^{*}(M, M)$ for all $k \leq k_{m}$.

Proof: Eq. (7) indicates that the volume sold by firm $i, i=1,2$, at the equilibrium $\{B, B\}$ is clearly decreasing in $k$ and then the equilibrium price $p_{i}^{*}(B, B)=1-q_{i}^{*}(B, B)-r q_{j}^{*}(B, B)(i, j=1,2$ and $i \neq j)$ is increasing in $k$, so the first part of Proposition 4 holds true.

Define $F_{i}:[0,1] \rightarrow \mathrm{R}, i=1,2$ as

$$
F_{i}(k)=q_{i}^{*}(B, B)-q_{i}^{*}(M, M)=\frac{1-k c}{2\left(1+\rho \sigma^{2} k^{2}\right)+r}-\frac{1-c}{2+r}
$$

Clearly, $F_{i}(k)$ is strictly decreasing in $k$ with $F_{i}(0)>0$ and $F_{i}(1)<0$. Thus there exists a $k_{c} \in(0,1)$ such that $F_{i}\left(k_{c}\right)=0, F_{i}(k)>0$ for all $k<k_{c}$ and $F_{i}(k)<0$ for all $k>k_{c}$.

Note that, if $k_{c}>k_{0},\{\mathrm{~B}, \mathrm{~B}\}$ is not a Nash equilibrium in the simultaneous-move case or a subgame perfect equilibrium when sequential move is assumed and, hence, it does not make much sense to discuss $p_{i}^{*}(B, B)$. Therefore, let $k_{m}=\min \left\{k_{c}, k_{0}\right\}$. Then, for any $k \leq k_{m}$, we have:

$$
\begin{aligned}
p_{i}^{*}(B, B)-p_{i}^{*}(M, M) & =1-q_{i}^{*}(B, B)-r q_{j}^{*}(B, B)-\left[1-q_{i}^{*}(M, M)-r q_{j}^{*}(M, M)\right] \\
& =-\left[q_{i}^{*}(B, B)-q_{i}^{*}(M, M)\right]-r\left[q_{j}^{*}(B, B)-q_{j}^{*}(M, M)\right] \leq 0
\end{aligned}
$$

This completes the proof of Proposition 4.

Remark 5: Propositions 2 and 4 clearly reveal a relationship between economies of scope and market prices when the firms' in-house production cost is high $\left(c>c_{0}\right)$ : if firms make their outsourcing decisions sequentially, E2 becomes the unique subgame perfect equilibrium of $G_{O}^{\prime}$ as long as the degree of scope economies exceeds the critical level $\left(k<k_{0}\right)$ and, eventually, market prices are driven lower as scope economies reach the level of $k_{m}$. Due to the uniqueness of the subgame perfect equilibrium E2, this prediction is deemed viable. Note that, if either firm chooses $\mathrm{M}$, its optimal volume and the corresponding payoff are independent of the degree of scope economies $(k)$, implying that the benefit of scope economies can only be realized through MCO. Propositions 2 and 4 provide a reasonable explanation for the emergence of MCO, resulting in the occurrence of supply chain cities and lowering market prices (e.g. the China price phenomenon). It is worth noting that the result here is significantly different from that in Cachon and Harker 
(2002). The equilibrium prices under scope economies here, $p_{i}^{*}(B, B)$, is lower than $p_{i}^{*}(M, M)$ when both firms in-source. However, Cachon and Harper (2002) predict an increase in final market prices under scale economies by collective outsourcing.

\section{Discussions}

\subsection{Risk-averse firms}

This subsection shows that Lemma1 and Proposition 1 through 4 still hold true for a wide spectrum of risk aversion profiles when firms are assumed to be risk-averse rather than risk-neutral as discussed earlier. This assumption of risk-averse firms may apply to the situation that firms are not so well diversified and, hence, have limited risk tolerance level as the vendor does. Now assume that both firms have the same risk aversion $\rho_{F} \geq 0 \quad$ ( $\rho_{F}=0$ corresponds to risk-neutral firms) and the same variance of production cost $\sigma_{F 1}^{2}=\sigma_{F 2}^{2}=\sigma_{F}^{2}$ where $\sigma_{F}^{2}$ is not necessarily equal to $\sigma^{2}$. The impact of the risk-averse assumption for a firm is that its cost increases when it chooses $M$. For instance, in the case of $\{M, M\}$, firm $i$ 's $(i=1,2)$ market decision can be rewritten as

$$
\pi_{i}^{*}(M, M)=\max _{q_{i}>0}\left\{\left(1-q_{i}-r q_{j}\right) q_{i}-c q_{i}-\frac{1}{2} \rho_{F} \sigma_{F}^{2} q_{i}^{2}\right\}
$$

where the last term in the objective function reflects the impact of the risk-aversion assumption.

For $\{M, B\}$ and $\{B, M\}$, the firms' market decisions can be analogically revised. But the firms' decisions remain unchanged for $\{\mathrm{B}, \mathrm{B}\}$, as neither firm incurs in-house production cost. Following the similar procedures in Sections 3 through 5, Proposition 5 is derived.

Proposition 5: If $\rho_{F} \sigma_{F}^{2}<2 \rho \sigma^{2}$, Lemma 1 and Propositions 1 through 4 hold true where the thresholds $c_{0}$, $k_{0}$ and $k_{m}$ shift with $\rho_{F}$ and $\sigma_{F}^{2}$.

Proof: First, the market equilibrium quantities and payoffs are obtained. It is clear that $q_{i}^{*}(B, B)$ and $\pi_{i}^{*}(B, B) \quad(i=1,2)$ remain. Other equilibrium variables are given below.

$$
\begin{gathered}
q_{i}^{*}(M, M)=\frac{1-c}{2+\rho_{F} \sigma_{F}^{2}+r}, \quad \pi_{i}^{*}(M, M)=\frac{2+\rho_{F} \sigma_{F}^{2}}{2}\left(\frac{1-c}{2+\rho_{F} \sigma_{F}^{2}+r}\right)^{2}(i=1,2) \\
q_{1}^{*}(B, M)=\frac{\left(2+\rho_{F} \sigma_{F}^{2}-r\right)(1-c)}{2\left(2+\rho_{F} \sigma_{F}^{2}\right)\left(1+\rho \sigma^{2}\right)-r^{2}}, \quad \pi_{1}^{*}(B, M)=\left(1+\rho \sigma^{2}\right)\left[\frac{\left(2+\rho_{F} \sigma_{F}^{2}-r\right)(1-c)}{2\left(2+\rho_{F} \sigma_{F}^{2}\right)\left(1+\rho \sigma^{2}\right)-r^{2}}\right]^{2}
\end{gathered}
$$

and

$$
q_{2}^{*}(B, M)=\frac{\left(2\left(1+\rho \sigma^{2}\right)-r\right)(1-c)}{2\left(2+\rho_{F} \sigma_{F}^{2}\right)\left(1+\rho \sigma^{2}\right)-r^{2}}, \quad \pi_{2}^{*}(B, M)=\frac{2+\rho_{F} \sigma_{F}^{2}}{2}\left[\frac{\left(2\left(1+\rho \sigma^{2}\right)-r\right)(1-c)}{2\left(2+\rho_{F} \sigma_{F}^{2}\right)\left(1+\rho \sigma^{2}\right)-r^{2}}\right]^{2}
$$

The symmetry of our model implies that $q_{1}^{*}(M, B)=q_{2}^{*}(B, M), \quad q_{2}^{*}(M, B)=q_{1}^{*}(B, M)$, $\pi_{1}^{*}(M, B)=\pi_{2}^{*}(B, M)$ and $\pi_{2}^{*}(M, B)=\pi_{1}^{*}(B, M)$. With this equilibrium result, following step-by-step the proofs of Lemma 1 and Propositions 1 through 4, Proposition 5 can be proved with shifted thresholds $c_{0}$, $k_{0}$ and $k_{m}$.

Remark 6: Proposition 5 indicates that the results under the risk-neutrality assumption remain valid even if the firms are risk averse as long as their risk costs caused by risk aversion are not too high $\left(\rho_{F} \sigma_{F}^{2}<2 \rho \sigma^{2}\right)$. Rearranging the terms yields $\rho_{F} / \rho<2 \sigma^{2} / \sigma_{F}^{2}$. So an alternative interpretation is that the conclusions of Lemma 1 and Propositions 1-4 remain true if the firms' risk aversion (relative to the vendor's) is not too severe. In the real business world, outsourcers (firms) tend to be large and diversified (sometimes, even publicly held) companies while suppliers (vendors) are often specialized and relatively small (sometimes, 
even privately owned) entities. As such, outsourcers are usually more capable of diversifying their investment than suppliers to reduce risk. Therefore, it is reasonable to assume that the degree of an outsourcer's risk aversion is lower (or at least, not much higher) than a supplier's. ${ }^{12}$ Therefore, this observation can be viewed as an empirical justification of the condition $\left(\rho_{F} \sigma_{F}^{2}<2 \rho \sigma^{2}\right)$. In the extreme case of $\rho_{F}=0$, firms become risk-neutral and the aforesaid arguments obviously hold.

\subsection{Asymmetric costs between firms}

This subsection relaxes the assumption of $E c_{v}^{1}=c_{1}=c=c_{2}=E c_{v}^{2}$ in Section 2 to a more general asymmetric cost case: $E c_{v}^{1}=c_{1}$ and $E c_{v}^{2}=c_{2}$ where $c_{1}$ is not necessarily equal to $c_{2}$. Below we show that Lemma 1, Propositions 1-2 and 4 hold true if $\left(c_{1}, c_{2}\right)$ satisfies certain conditions.

Let

$$
\begin{gathered}
A=\left\{\left(c_{1}, c_{2}\right): c_{i}>\frac{r \rho \sigma^{2}}{(2+r)\left(1+\rho \sigma^{2}\right)}+\frac{r c_{j}}{2\left(1+\rho \sigma^{2}\right)}, i \neq j \text { and } i, j=1,2\right\} \\
B=\left\{\left(c_{1}, c_{2}\right): c_{i}>\frac{r-2}{r}+\frac{2 c_{j}}{r}, i \neq j \text { and } i, j=1,2\right\} \\
C=\left\{\left(c_{1}, c_{2}\right): c_{i}>\frac{r c_{j}}{2}, i \neq j \text { and } i, j=1,2\right\}, D=(0,1) \times(0,1) \text { and } E=A \cap B \cap C \cap D
\end{gathered}
$$

Proposition 6: If $c>c_{0}$ is replaced with $\left(c_{1}, c_{2}\right) \in E$, where $c_{1}$ is not necessarily equal to $c_{2}$, Lemma 1, Propositions 1-2 and 4 hold true with shifted thresholds $k_{0}$ and $k_{m}$.

Proof: We first obtain the market equilibrium variables as follows:

$$
\begin{gathered}
q_{i}^{*}(M, M)=\frac{2\left(1-c_{i}\right)-r\left(1-c_{j}\right)}{4-r^{2}}, \pi_{i}^{*}(M, M)=\left[\frac{2\left(1-c_{i}\right)-r\left(1-c_{j}\right)}{4-r^{2}}\right]^{2}(i=1,2) \\
q_{i}^{*}(B, B)=\frac{2\left(1+\rho \sigma^{2} k^{2}\right)\left(1-k c_{i}\right)-r\left(1-k c_{j}\right)}{4\left(1+\rho \sigma^{2} k^{2}\right)^{2}-r^{2}}, \pi_{i}^{*}(B, B)=\left(1+\rho \sigma^{2} k^{2}\right)\left[\frac{2\left(1+\rho \sigma^{2} k^{2}\right)\left(1-k c_{i}\right)-r\left(1-k c_{j}\right)}{4\left(1+\rho \sigma^{2} k^{2}\right)^{2}-r^{2}}\right]^{2} \quad(i=1,2) \\
q_{1}^{*}(B, M)=\frac{2\left(1-c_{1}\right)-r\left(1-c_{2}\right)}{4\left(1+\rho \sigma^{2}\right)-r^{2}}, \pi_{1}^{*}(B, M)=\left(1+\rho \sigma^{2}\right)\left[\frac{2\left(1-c_{1}\right)-r\left(1-c_{2}\right)}{4\left(1+\rho \sigma^{2}\right)-r^{2}}\right]^{2} \\
q_{2}^{*}(B, M)=\frac{2\left(\left(1+\rho \sigma^{2}\right)\left(1-c_{2}\right)-r\left(1-c_{1}\right)\right.}{4\left(1+\rho \sigma^{2}\right)-r^{2}}, \pi_{2}^{*}(B, M)=\left[\frac{2\left(1+\rho \sigma^{2}\right)\left(1-c_{2}\right)-r\left(1-c_{1}\right)}{4\left(1+\rho \sigma^{2}\right)-r^{2}}\right]^{2} \\
q_{1}^{*}(M, B)=\frac{2\left(1+\rho \sigma^{2}\right)\left(1-c_{1}\right)-r\left(1-c_{2}\right)}{4\left(1+\rho \sigma^{2}\right)-r^{2}}, \pi_{1}^{*}(M, B)=\left[\frac{2\left(1+\rho \sigma^{2}\right)\left(1-c_{1}\right)-r\left(1-c_{2}\right)}{4\left(1+\rho \sigma^{2}\right)-r^{2}}\right]^{2} \\
q_{2}^{*}(M, B)=\frac{2\left(\left(1-c_{2}\right)-r\left(1-c_{1}\right)\right.}{4\left(1+\rho \sigma^{2}\right)-r^{2}}, \pi_{2}^{*}(M, B)=\left(1+\rho \sigma^{2}\right)\left[\frac{2\left(1-c_{2}\right)-r\left(1-c_{1}\right)}{4\left(1+\rho \sigma^{2}\right)-r^{2}}\right]^{2}
\end{gathered}
$$

\footnotetext{
${ }^{12}$ The diversification argument for explaining the difference of risk attitude is also cited in the classical agency theory (Eisenhardt 1989) and its applications (e.g. Chalos and Sung 1998).
} 
Now, for $\left(c_{1}, c_{2}\right) \in E$, it is easy to verify the following four results:

(I) $\pi_{1}^{*}(M, M)>\pi_{1}^{*}(B, M)$ and $\pi_{2}^{*}(M, M)>\pi_{2}^{*}(M, B)$.

(II) $\left(c_{1}, c_{2}\right) \in E \Rightarrow\left(c_{1}, c_{2}\right) \in A \Rightarrow\left\{\begin{array}{l}G_{1}(0)>0 \text { and } G_{1}(1)<0 \\ G_{2}(0)>0 \text { and } G_{2}(1)<0\end{array}\right.$,

where $G_{1}(k)=\pi_{1}^{*}(B, B)-\pi_{1}^{*}(M, B)$ and $G_{2}(k)=\pi_{2}^{*}(B, B)-\pi_{2}^{*}(B, M)$.

(III) $\left(c_{1}, c_{2}\right) \in E \Rightarrow\left(c_{1}, c_{2}\right) \in B \Rightarrow \pi_{1}^{*}(M, M)<\pi_{1}^{*}(M, B)$ and $\pi_{2}^{*}(M, M)<\pi_{2}^{*}(B, M)$.

(IV) $\left(c_{1}, c_{2}\right) \in E \Rightarrow\left(c_{1}, c_{2}\right) \in C \Rightarrow F_{i}(0)>0$ and $F_{i}(1)<0(i=1,2)$,

where $F_{i}(k)=q_{i}^{*}(B, B)-q_{i}^{*}(M, M)$.

(I) and (III) immediately imply that Lemma 1 and Proposition 2 hold, respectively. With the continuity of $G_{1}(k)$ and $G_{2}(k)$, (II) indicates that there exists a $k_{0}$ such that $(\mathrm{B}, \mathrm{B})$ is a Nash equilibrium of the MOB game for all $k<k_{0}$. Here, $k_{0}$ is defined as the minimal $k$ such that $G_{1}(k)=0$ or $G_{2}(k)=0$ since $G_{1}(k)$ and $G_{2}(k)$ are not necessarily monotonic. Thus, Proposition 1 holds. Further, (IV) implies that there exists a $k_{c}$ such that $F_{i}(k)>0$ for all $k<k_{c}$, where $k_{c}$ is defined as the minimal $k$ such that $F_{1}(k)=0$ or $F_{2}(k)=0$, due to the non-monotonicity of $F_{1}(k)$ and $F_{2}(k)$. Let $k_{m}=\min \left\{k_{c}, k_{0}\right\}$, then Proposition 4 follows.

Finally we show that there exists some $\left(c_{1}, c_{2}\right) \in E$ such that $c_{1} \neq c_{2}$. In fact, for all $c \in\left(c_{0}, 1\right)$, $\left(c_{1}, c_{2}\right)=(c, c) \in E$. Since $E$ is open, there exists a neighborhood $U((c, c)) \subset E$ which contains some $\left(c_{1}, c_{2}\right) \in U((c, c)) \subset E$ satisfying $c_{1} \neq c_{2}$. The proof of Proposition 6 is completed.

Remark 7: Proposition 6 demonstrates that Lemma 1, Propositions 1-2 and 4 can be extended to a more general asymmetric cost case. This result confirms that scope economies can still be regarded as a motivation for firms to outsource collectively as long as the difference between $c_{1}$ and $c_{2}$ is not too significant. More specifically, for a given $c_{1} \in\left(c_{0}, 1\right)$, if

$$
\max \left\{\frac{r \rho \sigma^{2}}{(2+r)\left(1+\rho \sigma^{2}\right)}+\frac{r c_{1}}{2\left(1+\rho \sigma^{2}\right)}, \frac{r-2}{r}+\frac{2 c_{1}}{r}, \frac{r c_{1}}{2}\right\}<c_{2}<\min \left\{\frac{2\left(1+\rho \sigma^{2}\right) c_{1}}{r}-\frac{2 \rho \sigma^{2}}{2+r}, \frac{r c_{1}}{2}-\frac{r-2}{2}, \frac{2 c_{1}}{r}\right\},
$$

then there exist thresholds $k_{0}$ and $k_{m}$ such that the conclusions of Lemma 1, Propositions 1-2 and 4 remain true. However, Proposition 3 cannot be extended so naturally. Note that the non-monotonicity of $G_{i}(k)$ implies that $W_{i}(k)$ (defined as $W_{1}(k)=\sqrt{\pi_{1}^{*}(B, B)}-\sqrt{\pi_{1}^{*}(M, B)}$ and $W_{2}(k)=\sqrt{\pi_{2}^{*}(B, B)}-\sqrt{\pi_{2}^{*}(B, M)}$ ) may not be monotonic, indicating that $W_{i}(k)=0$ may have multiple solutions. Therefore, the implicit functional relationship (specified by $\left.W_{i}(k)=0\right)$ among $k, r, c_{1}, c_{2}, \rho$ and $\sigma^{2}$ may not be well defined. Note further that $G_{i}(k)>(=,<) 0$ is equivalent to $W_{i}(k)>(=,<) 0$ and $k_{0}$ is the minimal $k$ such that $W_{1}(k)=0$ (or $G_{1}(k)=0$ ) or $W_{2}(k)=0$ (or $G_{2}(k)=0$ ). Without loss of generality, let $W_{1}\left(k_{0}\right)=0$. Then for all $\left(c_{1}, c_{2}\right) \in E, r, \rho$ and $\sigma^{2}, \partial W_{1} /\left.\partial k\right|_{k=k_{0}} \leq 0$ by $W_{1}(0)>0$ and $k_{0}$ is the minimal $k$ such that $W_{1}(k)=0$ (that is, at $k_{0}$, it is the first point where $W_{1}(k)$ crosses the k-axis from positive to negative, or be tangent to the $k$-axis). However, we cannot generally determine the signs of $\partial W_{1} / \partial c_{1}, \quad \partial W_{1} / \partial c_{2}, \partial W_{1} / \partial r, \partial W_{1} / \partial \rho$ and $\partial W_{1} / \partial \sigma^{2}$. The reason is that, compared to the symmetric cost case, the cost difference brings additional non-zero components in the expressions of $\partial W_{1} / \partial r$, $\partial W_{1} / \partial \rho$ and $\partial W_{1} / \partial \sigma^{2}$ on the one hand, and on the other hand, non-zero components (which are different from those in $\partial W_{1} / \partial r, \partial W_{1} / \partial \rho$ and $\left.\partial W_{1} / \partial \sigma^{2}\right)$ are absent from the expressions of $\partial W_{1} / \partial c_{1}$ and $\partial W_{1} / \partial c_{2}$. Therefore, Proposition 3 cannot be naturally extended to the cost-asymmetric case. Nevertheless, the logic in the proof of Proposition 3 can be applied to examine the comparative statics of $k_{0}$ 
with respect to $c_{1}, c_{2}, r, \rho$ and $\sigma^{2}$ by using appropriate simulation technologies.

\section{Concluding Remarks}

This paper develops two game models based on production costs and scope economies to investigate the widely observed MCO phenomenon. Main analytical results include: (1) if the degree of scope economies is low or in-house production costs are low, it is impossible for both firms to outsource collectively (Corollary 1); (2) if they reach sufficiently high levels $\left(c>c_{0}\right.$ for the symmetric cost case or $\left(c_{1}, c_{2}\right) \in E$ for the asymmetric cost case, and $k<k_{0}$ ) and both firms move simultaneously, collective outsourcing is on an equilibrium path (E2) of $G_{O}$, exhibiting a possibility of MCO (see Lemma 1 and the summary of Section 3); (3) if scope economies and in-house production exceed their threshold levels and both firms make their outsourcing decision sequentially, collective outsourcing becomes the unique perfect equilibrium of the modified MOB game and, then, E2 arises as the unique subgame perfect equilibrium of $G_{O}^{\prime}$ (see Proposition 2), implying that MCO is a viable prediction in the presence of high enough in-house production costs and strong enough scope economies. As supply chain cities can be viewed as an outcome of MCO, our results thus help account for the emergence of supply chain cities.

Moreover, Proposition 3 examines the comparative statics of the critical degree of scope economies $\left(k_{0}\right)$ with regard to the market parameter $(r)$, firms' technology parameter $\left(c \in\left(c_{0}, 1\right)\right.$, the vendor's risk attitude $(\rho)$, and the exogenous uncertainty $\left(\sigma^{2}\right)$. These results are valuable for practitioners to anticipate how the critical degree of scope economies changes as those parameters change.

Finally, it is proved that market prices decrease as the degree of scope economies increases in the presence of MCO and market prices under MCO are lower than those when both firms produce in-house within a certain range of scope economies. The empirical implication is that MCO can serve as a means to exploiting scope economies and, then, leading marker prices lower even if both in-house and the vendor's production costs are high. Therefore, this result facilitates us to understand the universally- observed "China price" phenomenon.

Significant work remains open. The current model considers only two firms versus one vendor with relatively homogeneous productivities. However, in reality, it is often the case that $n(n>2)$ firms exist in the same industry with heterogeneous productivity profiles. In addition, multiple vendors may compete for outsourcing firms in the marketplace as well. As such, it is worthwhile to extend the model here to accommodate more complicated cases such as $n(n>2)$ firms versus one vendor and $n(n>2)$ firms versus $m$ $(m>1)$ vendors with diverse productivity levels.

Another meaningful extension is to introduce agency costs arising from information asymmetry into our model and this extension should be able to explore more thoroughly the relationships among production costs, scope economies, MCO, and market prices. Still a different line of research is to interpret the source of scope economies as more concrete business terms such as social networks, public operational platforms, integrated knowledge, and innovation incentive mechanisms, to name a few. All of these open problems warrant further investigations.

\section{Acknowledgments}

The authors wish to express their appreciation to the two anonymous reviewers for their constructive comments that significantly improve the quality and presentation of the paper. Debing Ni would like to acknowledge the financial support from the Natural Sciences Foundation (NSF) of China (Grant \#: 70702025) and the UESTC Funds for Junior Research Chairs. Kevin W. Li is grateful for the financial support from the Natural Sciences and Engineering Research Council of Canada (NSERC) under the 
Discovery Grant program and the International \& Development Research, Education \& Training (IDRET) Seed Monies Grant from the University of Windsor.

\section{References}

[1] Bailey, E. E. and Friedlaender, F. (1982). Market structure and multiproduct industries. Journal of Economic Literature, 20(3): 1024-1048

[2] Berglund, M., van Laarhoven, P. , Sharman, G., and Wandel S. (1999), Third-party logistics: Is there a future? International Journal of Logistics Management, 10(1): 59-70.

[3] Bhattacharya, A., Bradtke, T., Hemerling, J., Lebreton, J., Mosquet, X., Rupf, I., Sirkin, H. L, .and Young. D. (2004). Capturing global advantage: How leading industrial companies are transforming their industries by sourcing and selling in China, India, and Other Low-Cost Countries. Boston Consulting Group Report, April

[4] Cachon, G. P. and Harker, P. T. (2002). Competition and outsourcing with scale economies. Management Science, 48(10): $1314-1333$

[5] Cheung, S. (1983).The contract nature of the outsourcer. Journal of Law and Economics, 26(1): 1-21

[6] Chalos, P. and Sung, J. (1998). Outsourcing decisions and managerial incentives. Decision Sciences, 29(4): 901-919

[7] Coase, R.H.. (1973). The nature of the outsourcer. Economica, 4(16): 368-405

[8] Dibbern, J., Goles, T, Hirschheim, R., and Jayatilaka, B. (2004). Information systems outsourcing: A survey and analysis of the literature. DATA BASE for Advance in Information Systems, 35(4): 6-102

[9] Dube, P., Liu, Z., Wynter, L. and Xia, C. (2007). Competitive equilibrium in e-commerce: Pricing and outsourcing. Computers \& Operations Research, 34(12): 3541-3559

[10] Eisenhardt, K. M. (1989). Agency theory: An assessment and review. Academy of Management Review, 14(1): 57-74

[11] Engardio, P., Roberts, D. and Bremner, B. (2004). The China price. Business Week, Dec. 6: 102-112

[12] Gallivan, M. J. and Oh, W. (1999). Analyzing IT outsourcing relationships as alliances among multiple clients and vendors. Proceedings of the 32nd Hawaii International Conference on System Sciences, 1999

[13] Gordon, I. R. and McCann, P. (2000). Industrial clusters: complexes, agglomeration and/or social networks? Urban Studies, 37(3): 513-532

[14] Grossman, S. and Hart, O. (1986). The costs and benefits of ownership: A theory of vertical and lateral integration. Journal of Political Economy, 94(4): 691-719

[15] Hart, O. and Moore, J. (1990). Property rights and the nature of the outsourcer. Journal of Political Economy, 98(6): 1119-1158

[16] Kahn, G. (2004). Making labels for less. Wall Street Journal, August 13: B1-B3

[17] Kusterbeck, S. (2005), China appeals to U.S. buyers with 'supply chain cities'. Apparel Magazine, 46(12): 24-28

[18] Morosini, P. (2004). Industrial clusters, knowledge integration and performance. World Development, 32(2): $305-326$

[19] Osei-Bryson, K-M. and Ngwenyama, O. K. (2006). Managing risks in information systems outsourcing: An approach to analyzing outsourcing risks and structuring incentive contracts. European Journal of operational Research, 174(1): 245-264

[20] Panzar, J. C. and Willig, R. D. (1977). Economies of scale in multi-output production. Quarterly Journal of Economics, 91(3): 431-493

[21] Panzar, J. C. and Willig, R. D. (1981). Economies of scope. American Economic Review, 71(2): 268-272

[22] Porter, M. E. (1998). Clusters and the new economics of competition. Harvard Business Review, 76(6): 77-90

[23] Sharma, R. and Yetton, P. (1996). Interorganizational cooperation to develop information systems. Proceedings of the $17^{\text {th }}$ International conference on information systems, 122-132

[24] Sridhar, S. S. and Balachandran, B. V. (1997). Incomplete information, task assignment, and managerial control systems. Management Science, 43(6): 764-778 
[25] Stefansson, G. (2002). Business-to-business data sharing: A source for integration of supply chains, International Journal of Production Economics, 75(1-2): 135-146.

[26] van Mieghem, J. (1999). Coordinating investment, production and subcontracting. Management Science, 45(7): 954-971

[27] Vega-Redondo, F. (2003). Economics and the Theory of Games. Cambridge: Cambridge University Press.

[28] Whang, S. (1992). Contracting for software development. Management Science, 38(3): 307-324

[29] Williamson, O. E. (1975). Markets and Hierarchies: Analysis and Antitrust Implications. New York: Free Press

[30] Wu, L. Yue, X. and Sim, T. (2006). Supply chain clusters: A key to China's cost advantage. Supply Chain Management Review, 10(2): 46-51 